\title{
Über den Einfluß der tropistischen Reizung auf den Stoffwechsel der gereizten Organe, I.
}

Der Zuckergehalt und die Atmungsintensität geotropisch gereizter Sprosse

\author{
Von Hubert Ziegler
}

\author{
Aus dem Botanischen Institut der Universität München \\ (Z. Naturforschg. 6 b, 200-206 [1951]; eingegangen am 20. März 1951)
}

\begin{abstract}
1. Die bereits mehrfach festgestellte Zuckerdifferenz zwischen der Unter- und Ober- bzw. Konvex- und Konkavseite geotropisch gereizter Sprosse setzt mit der Aufkrümmung ein und geht nach Beendigung der Reaktion wieder vollständig zurück.

2. Die geotropische Reizung führt bei Sprossen zu einer stärkeren Sauerstoffaufnahme der Unter- bzw. Konvexseite gegenüber der Ober- oder Konkavseite. Es handelt sich dabei um eine Intensivierung der Atmung.

3. Die Ausbildung der Atmungsdifferenz wird gleichzeitig mit der Krümmung und dem Auftreten der Zuckerunterschiede deutlich. Auch hier kommt es nach Beendigung der Reaktion wieder zu einem vollständigen Ausgleich.

4. Die beobachteten Unterschiede in den Atmungsintensitäten sind nur zu einem geringen Teil auf die Zuckerdifferenzen zurückzuführen.

5. Der mögliche Zusammenhang mit der bei der Reizung stattfindenden Wuchsstoffverschiebung wird diskutiert.
\end{abstract}

Tricti rotz der außerordentlich zahlreichen Arbeiten, die sich mit den Ursachen und dem Mechanismus der tropistischen Reaktionen befassen, sind wir noch nicht in der Lage, uns ein klares Bild von der Art der durch den Reiz reversibel induzierten physiologischen Dorsiventralität zu machen, welche die Flanken des gereizten Organes derart im Wachstum beeinflußt, daß schließlich die Bedingungen für die physiologische Symmetrie wiederhergestellt werden.

Eine entscheidende Bedeutung in diesem Zusammenhang wird seit den Untersuchungen von $\mathrm{Cho-}$ $\operatorname{lodny^{1}}$ und Went ${ }^{2}$ dem Wuchsstoff zugeschrieben, dessen Bildung, Beständigkeit, Transport und wahrscheinlich auch Aktivität durch die Reize beeinflußt werden. Sollte unter den sicher vielfältigen Wirkungen des Lichtes und der Schwerkraft ${ }^{3}$, die als Reizursachen hauptsächlich in Betracht kommen, tatsächlich diese Veränderung des aktiven Wuchsstoffgehaltes ausschlaggebend sein, so ist die Frage nach dem Mechanismus der Reizkrümmungen aufs engste verknüpft mit dem Problem der Wirkungsweise des

1 N. C h o lo d n y, Ber. dtsch. bot. Ges. 42, 356 [1924]; Jb. wiss. Bot. 65, 447 [1926].

2 F. W. W e n t, Recueil Trav. bot. néerl. 25, 1 [1928].

3 H. Z i e g l e r, Planta 38, 474 [1950].

4 J. Bonner u. S. W. Wildmann, Growth 11, 51 [1941]. 1879 .
Wuchsstoffes in der Zelle, das in den letzten Jahren erfolgversprechend in Angriff genommen wurde. Es konnte dabei vor allem wahrscheinlich gemacht werden, daß der Wuchsstoff als Bestandteil eines Fermentes seine wachstumsfördernde Wirkung über eine Beeinflussung des Stoffwechsels geltend macht ${ }^{4}$.

Es liegt in diesem Zusammenhang nahe, nach den Stoffwechseldifferenzen zu fragen, die zwischen den Flanken eines tropistisch gereizten Organes auftreten, und zu prüfen, ob diese sich mit der Verschiebung des aktiven Wuchsstoffgehaltes in Verbindung bringen lassen. In der Literatur findet sich eine Reihe von Angaben, die von chemischen Veränderungen bei tropistischer Reizung berichten; so wurden bei der Unterseite geotropisch gereizter Sprosse ein etwas höherer Wassergehalt ${ }^{5,6,7}$, verminderte potentielle Acidität ${ }^{8,9,7}$, gesteigerte aktuelle Acidität ${ }^{10,11,12}$, eine Vermehrung der reduzierenden Zucker ${ }^{7}{ }^{12}$, eine verringerte Katalaseaktivität ${ }^{11}$, eine geringe Erhöhung des spezifischen Gewichtes und eine schwache Minderung der Leitfähigkeit ${ }^{12}$ gegenüber den entsprechen-

6 Th. G. Phillips, Bot. Gaz. 69, 168 [1920].

7 Th. W a r n e r, Jb. wiss. Bot. 68, 431 [1928].

8 G. Kra u s, Abh. Naturforsch. Ges. Halle 15, 49 [1882].

9 E. O. S c h le y, Bot. Gaz. 56, 480 [1913].

10 D. Greenwood u. W. H. Pearsall, Proc. Leeds philos. lit. Soc., sci. Sect. 2, 87 [1926].

11 W. G u n d e l, Jb. wiss. Bot. 78, 623 [1933].

12 P. M etz ner, Ber. dtsch. bot. Ges. 52, 506 [1934]. 
den Werten der Oberseite festgestellt. Bei horizonal gelegten Wurzeln fand $\mathrm{Kraus}{ }^{5}$ ebenfalls einen etwas erhöhten Wassergehalt der Unterseite. Phototropisch gereizte Sprosse ließen eine geringe Steigerung des Wassergehaltes ${ }^{5}$, eine erhöhte aktuelle Acidität und eine verstärkte Katalaseaktivität der Schattenflanke erkennen ${ }^{13}$, dagegen keine verwertbare Verschiedenheit im Gehalt an reduzierenden Zuckern ${ }^{8,13}$.

Sind die eben geschilderten Befunde wenigstens von einem Teil der zitierten Autoren auf methodisch einwandfreiem Wege erhalten. (Warner, Gun del, M e tz n er) und daher nicht zu bezweifeln, so trifft dies für die Angaben von $\mathrm{S} \mathrm{chle} \mathrm{y}{ }^{9}$ über verstärkten Turgor, gesteigerte Atmung und Verminderung der Polysaccharide auf der Konvexseite geotropisch gereizter Sprosse, von $\mathrm{Ph}$ illip s ${ }^{6}$ über ein Fehlen der Unterschiede in der Katalaseaktivität, dem Säure- und dem Zuckergehalt bei geotropischer Reizung, von $\mathrm{Grafe}$ und $\mathrm{Lins} b$ a u e ${ }^{14}$, die keine Differenzen der aktuellen Acidität geotropisch gereizter Keimwurzeln erhielten, von $\mathrm{G}$ ree $\mathrm{n}$ w o od und $\mathrm{Pears}$ all10 über nicht einheitliche Schwankungen des $p_{\mathrm{H}}$-Wertes bei verschiedenen geotropisch gereizten Wurzeln und Sprossen ebensowenig zu wie für den alten $\mathrm{C} \mathrm{z}$ a p e k schen Befund einer vermehrten Bildung eines Hydrochinonderivates, der „Homogentisinsäure", in tropistisch gereizten Organen ${ }^{15}$.

Die einzige gesicherte chemische Differenz, welche die Konvexflanken sowohl der geisch wie der photisch gereizten Sprosse gegenüber den Konkavseiten gemeinsam aufweisen, ist demnach, worauf auch Metz $\mathrm{n}$ e $\mathrm{r}^{\mathbf{1 3}}$ hinweist, eine Erhöhung der aktuellen Acidität. Nun bestehen zwischen der Acidität des Protoplasmas und dem Streckungswachstum sicher Beziehungen ${ }^{16}$, doch sind sie vorläufig nicht so klar, daß sie wesentlich zur Lösung unseres Problems beitragen könnten. Es darf auch nicht vergessen werden, daß bei allen erwähnten Bestimmungen stets nur der $p_{\mathrm{H}}$-Wert eines ganzen Zellverbandes erfaßt wurde, der keinen Schluß auf die Verhältnisse in den für das Wachstum entscheidenden plasmatischen Bezirken der einzelnen Zellen zuläßt. Unklar ist schließlich auch, ob zwischen den Aciditätsverschiebungen und den Schwankungen im Wuchsstoffgehalt ein direkter Zusammenhang besteht.

Waren demnach die Bemühungen, aus der näheren

13 P. M etz n e r, Ber. dtsch. bot. Ges. 54, 455 [1936].

14 V. Grafe u. K. Lins bauer, 1. u. 2. Sitz.-B. Akad. Wiss. Wien, math.-nat. Kl. I, 118, 907 [1909], und 119, 827 [1910].
Kenntnis der Änderungen im Chemismus der gereizten Organe tiefere Einblicke in den Mechanismus der tropistischen Krümmungen zu gewinnen, wenig erfolgreich, so dürfte dies vor allem daran liegen, daß bisher nicht eigentliche Stoffwechselprozesse erfaßt wurden. Die einzige entsprechende Angabe stammt

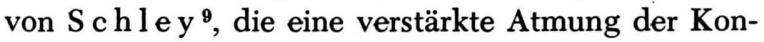
vexseite geotropisch gereizter Sprosse feststellen zu können glaubte. Dieser Befund ist jedoch von geringer Beweiskraft, weil er nur qualitativ erhalten wurde und vor allem weder Frisch- noch Trockengewicht der geprüften Einzelflanken angegeben wurden.

Da nun aber die heutige Vorstellung von der Rolle des Wuchsstoffes durchaus vermuten läßt, daß eine einseitige Verschiebung $\mathrm{zu}$ faßbaren Änderungen des Atmungsstoffwechsels führen könnte, soll diese Frage eingehend untersucht werden. Die vorliegende Arbeit berichtet zunächst über die Beeinflussung der Atmungsintensität von Sprossen durch eine geotropische Reizung und den Zusammenhang mit den bekannten Zuckerdifferenzen. Eine Darstellung der Verhältnisse bei positiv geotropischen und bei positiv und negativ phototropischen Organen sowie eine eingehendere Analyse der Grundlagen der Stoffwechseländerungen und eine Klärung der Beziehungen zu der Wuchsstoffverschiebung soll weiteren Arbeiten vorbehalten bleiben.

\section{Die Versuche}

A. Material und allgemeine Methodik

Die Versuche wurden mit 8 Wochen alten Sprossen von Helianthus annuus und $1 / 2$-jährigen Sprossen von Bryophyllum tubiflorum durchgeführt. Die Pflanzen waren im Gewächshaus bei etwa $20^{\circ} \mathrm{C}$ gewachsen.

Zur geotropischen Reizung wurden sie in einer Dunkelkammer bei $30^{\circ}\left(\mp 1^{\circ} \mathrm{C}\right)$ horizontal gelegt. Bei der Feststellung der Reaktionszeit und des Reaktionsverlaufes wurde bei Rotlicht zunächst die Krümmung mit dem Horizontalmikroskop verfolgt und das weitere Fortschreiten auf Millimeterpapier nachgezeichnet. Nach Beendigung der Reizung wurden die Sprosse mittels einer Rasierklinge in möglichst gleiche Hälften gespalten.

Bei den Zuckerbestimmungen wurde zuerst das Frischgewicht der einzelnen Seiten bestimmt, diese dann in Porzellanmörsern mit reinem Quarzsand vollständig zerrieben, mit kochendem dest. Wasser übergossen, der Brei auf einen Trichter überführt und nochmals mit kochendem dest. Wasser durchgespült, wobei das Waschwasser des gründlich gereinigten Mörsers und Pistills mitverwendet wurde. Das Filtrat wurde in einem Meßkolben auf-

15 Fr. C z a pe k u. R. B e rt e l, Jb. wiss. Bot. 43, 361 [1906].

16 S. S tru g g e r, Jb. wiss. Bot. 79, 406 [1934]. 
genommen und nach dem Erkalten auf $50 \mathrm{ccm}$ aufgefüllt.

Diese Methode erlaubt einerseits, wie eine Prüfung des Rückstandes ergab, eine vollständige Gewinnung des Zuckers und stoppt andererseits die Fermenttätigkeit, so daß keine weitere Veränderung im Zuckergehalt des Extraktes stattfinden kann.

Die Zuckerbestimmung selbst erfolgte elektrometrisch ${ }^{17}$. Bei dieser Methode reduziert der Zucker zunächst eine citratalkalische Kupferlösung. Das Kupferoxydul wird mit Ferrisalz in salzsaurer Lösung direkt oxydiert und das entstandene Ferrosalz mit Kaliumbromat titriert, wobei der Potentialsprung in der Ladung einer in die Lösung eintauchenden Platinelektrode die Beendigung der Reaktion $\mathrm{Fe}^{++} \rightarrow \mathrm{Fe}^{+++}$anzeigt. Die Potentialmessung erfolgte mittels eines Röhrenvoltmeters nach Dr. B. L a n ge ; der Potentialsprung führte von etwa $500 \mathrm{mV}$ bis gegen $800 \mathrm{mV}$. Zur Titration wurde ein automatisches Titriergestell mit elektrischem Rührwerk verwendet. Je nach der Zuckerkonzentration, die in einem Vorversuch mit der gröberen Methode annähernd ermittelt wurde, gelangte teils die Halbmikromethode (6-24 mg Glucose in $50 \mathrm{ccm}$ ), teils die Mikromethode $(2,4-9,0 \mathrm{mg}$ Glucose in $50 \mathrm{ccm})$ zur Anwendung. Stets wurden von jedem Ansatz $3 \mathrm{Be}-$ stimmungen und 2 Leerbestimmungen durchgeführt.

Die elektrometrische Methode hat für die Zuckerbestimmung in Pflanzenextrakten den großen Vorteil, daß etwa vorhandene Trübungen nicht entfernt $\mathrm{zu}$ werden brauchen, da ja keinerlei optische Beurteilung vonnöten ist. Im übrigen ist sie bequem durchzuführen und sehr genau; eine Serie von 10 eingewogenen Kontrollen ergab eine mittlere Abweichung von 1,2\%.

Der Sauerstoffverbrauch wurde manometrisch bestimmt. Die Sproßhälften wurden dazu in etwa $2,5 \mathrm{~cm}$ lange Stücke zerschnitten und so in die Gefäße gebracht, die mit $3 \mathrm{ccm} \mathrm{1/45-mol.} \mathrm{Phosphatpuffers} \mathrm{(prim./sek.} \mathrm{nach}$ $\mathrm{S}$ örense n) vom $p_{\mathrm{H}}$ 6,4 beschickt waren. Im Gasraum befand sich Luft. Absorbiert wurde mit 0,3 ccm 1- $n$. Kalilauge. Die Versuchstemperatur betrug $30^{\circ} \mathrm{C}$. Um eine Assimilation auszuschalten, wurde durch Umwickeln der Gefäße mit schwarzem Tuch ein Lichtzutritt verhindert. Der Sauerstoffverbrauch wurde stets auf $1 \mathrm{mg}$ Trockengewicht bezogen. Über weitere Einzelheiten der Methode sowie über die Feststellung des respiratorischen Quotienten vergleiche $\mathbf{Z}$ i e $\mathrm{g}$ l e r $\mathbf{1 8}$.

\section{B. Versuchsergebnisse}

1. Der Verlauf der geotropischen Reaktion der verwendeten Sprosse

Einen typischen Krümmungsverlauf für HelianthusSprosse gibt Abb. 1 wieder. Die Reaktion beginnt nach $45 \mathrm{Min}$. und erreicht nach etwa $14 \mathrm{Stdn}$. einen Wert von $90^{\circ}$.

Weit träger wird die Aufrichtung bei Bryophyllum tubiflorum durchgeführt (Abb. 2); sie beginnt nach

17 E. M is lowitzer, Biochem. Z. 168, 217 [1925]; G. K le in, Handbuch der Pflanzenanalyse II/I, Wien 1932.

18 H. Z i e g l e r, Z. Naturforschg. 5 b, 345 [1950].
3 Stdn. mit einer Hebung der Gipfelknospe und erfaßt nach etwa 6 Stdn. die obersten Teile des Sprosses, für welche die Kurve gezeichnet wurde. Die Ver-. tikale wird nach etwa 24 Stdn. erreicht.

Beide Abbildungen zeigen jeweils die Reaktion eines Sprosses, deren Verlauf mit den Mittelwerten aus 5 Bestimmungen weitgehend übereinstimmt. Die Extreme lagen bei der Reaktionszeit für Helianthus bei 40 und 60 Min., für Bryophyllum, bei 5 und

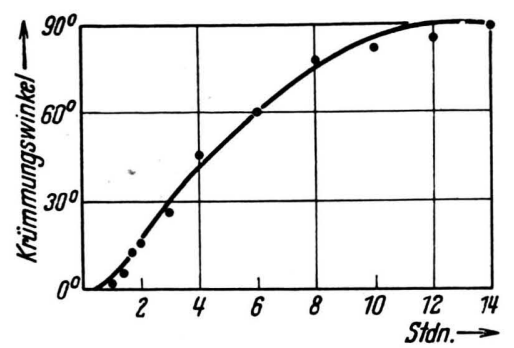

Abb. 1. Verlauf der geotropischen Krümmung der Sprosse von Helianthus annuus.

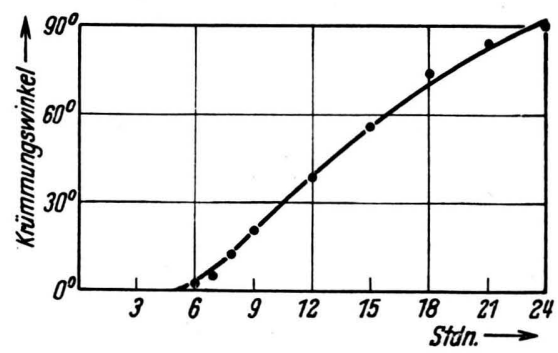

Abb. 2. Verlauf der geotropischen Krümmung der Sprosse von Bryophyllum tubiflorum.

6 $1 / 2$ Stdn., für das Ende der Krümmung für Helianthus bei 12 und 19 Stdn., für Bryophyllum bei 21 und 26 Stunden.

2. Die Zuckerdifferenzen $z$ wischen Sproßunter-und-oberseite während der Reaktion

Es muß zunächst betont werden, daß mit der geschilderten elektrometrischen Bestimmungsmethode nicht nur reduzierende Zucker erfaßt werden, sondern alle Substanzen, die zweiwertige Kupferionen in alkalischem Medium bei Erhitzung zu reduzieren vermögen. Da aber nach $\mathrm{Metzner}{ }^{12}$, der Helianthus-Sprosse untersuchte, der weit überwiegende Teil der in Pflanzenextrakten vorhandenen, entsprechend reduzierenden Substanzen aus Zuckern besteht, wird im folgenden der Reduktionswert stets vollständig auf Glucose umgerechnet und nur von „Zucker“Gehalt und „Zucker“-Differenzen gesprochen. 


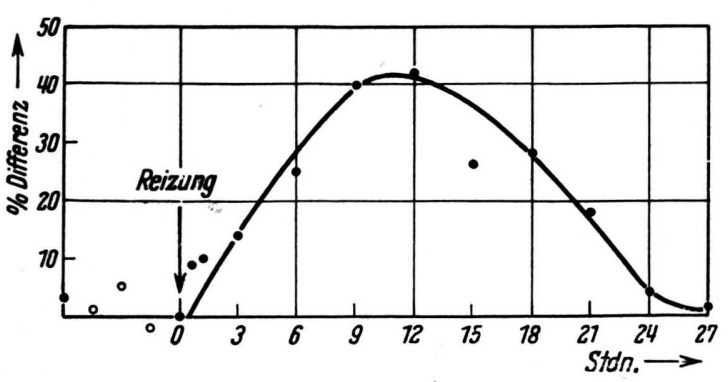

Abb. 3. Die Differenz im Zuckergehalt zwischen der Unterbzw. Konvex- und der Ober- bzw. Konkavseite geotropisch gereizter Helianthus-Sprosse in Prozent des jeweiligen Mittelwertes der beiden Hälften. $O=$ Differenz zwischen den Hälften ungereizter Sprosse.

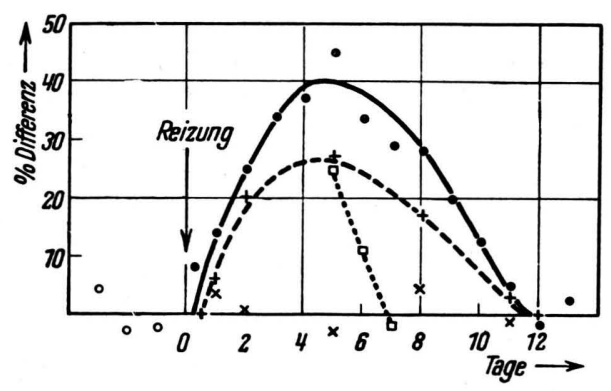

Abb. 4. Die Differenz im Zuckergehalt zwischen der Unterbzw. Konvex- und der Ober- bzw. Konkavseite der verschiedenen Sproßzonen von Bryophyllum tubiflorum in Prozent des jeweiligen Mittelwertes der beiden Hälften. $0=$ Differenz zwischen den Hälften ungereizter Sprosse. $\square----\mathrm{a}=$ Vertikalzone, $+--+=$ Horizontalzone, $\bullet-\bullet=$ Krümmungszone.

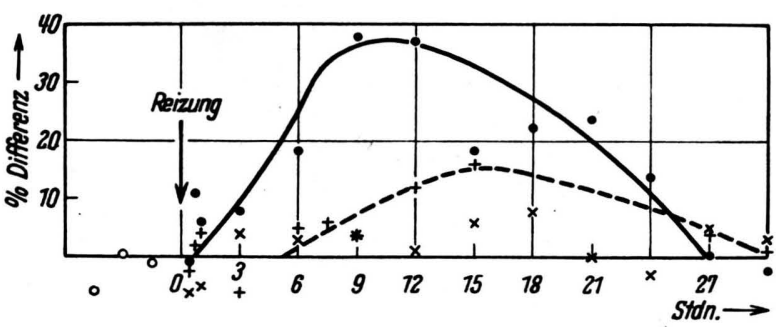

Abb. 5. Die Differenz im Sauerstoffverbrauch zwischen der Unter- bzw. Konvex- und der Ober- bzw. Konkavseite bei verschiedenen Abschnitten geotropisch gereizter HelianthusSprosse im Durchschnitt der ersten 6 Stdn. nach der Halbierung und in Prozenten des jeweiligen Mittelwertes der beiden Hälften. $\bullet-\bullet=$ Krümmungszone, $+\cdots+=$ obere Horizontalzone, $\mathrm{x}=$ untere Horizontalzone, $\mathrm{o}=$ Differenz zwischen den Hälften ungereizter Sprosse.

Wie erwähnt, wurde schon mehrfach festgestellt, daß die Unterseite geotropisch gereizter Sprosse einen höheren Zuckergehalt aufweist als die Oberseite. Nach den Angaben Warners ${ }^{7}$, der auch tagelang gereizte Sprosse untersuchte, setzt die Differenz einige Zeit nach Beginn der Krümmung ein, nimmt mit der
Reizdauer zu und zeigt z. B. für Dahlia auch nach 14 Tagen noch keinerlei rückläufige Tendenz. Diese Befunde konnten nicht bestätigt werden; wie Abb. 3 für Helianthus und Abb. 4 für Bryophyllum zeigen, treten bei beiden Objekten die ersten faßbaren Zuckerunterschiede bei Beginn der Reaktion (Abb. 1 und 2) ein und klingen die Differenzen einige Zeit nach Beendigung der Krümmung wieder vollständig ab. In allen untersuchten Fällen wies aber - in Übereinstimmung mit den zitierten Angaben - die Unter- bzw. Konvexseite während der Reaktion einen höheren Zuckergehalt auf als die Ober- bzw. Konkavseite.

Das Maximum der Zuckerunterschiede wird bei Helianthus etwa bei der Zeit erreicht, wenn das Ende des gekrümmten Sprosses die Vertikale erreicht hat; von diesem Zeitpunkt an sinkt der Wert der Differenz stetig bis zum vollständigen Ausgleich ab.

Etwas anders liegen die Verhältnisse bei Bryophyllum; hier lassen sich zwar auch die ersten Zuckerunterschiede ungefähr bei Beginn der Aufrichtung fassen, doch sind sie erst nach 5 Tagen maximal ausgebildet, also lange nachdem der Sproß sich in die Vertikale eingekrümmt hat. Erst nach etwa 12 Tagen weisen Ober- und Unterseite wieder denselben Zuckergehalt auf. Krümmungszone und anschließender Horizontalabschnitt zeigen dabei eine im wesentlichen übereinstimmende Reaktion; allerdings sind die Zuckerunterschiede in den gekrümmten Teilen am stärksten ausgeprägt. In den weiter zum Sproßgrund gelegenen Zonen läßt der Zuckergehalt der Ober- und Unterseite keinerlei Abhängigkeit von der Reizung mehr erkennen, während bei den aus der Reizlage in die Vertikale eingekrümmten Sproßabschnitten die Differenz zunächst noch gut faßbar ist, dann aber sehr schnell abnimmt.

Die Absolutwerte des Zuckergehaltes schwanken im übrigen bei den einzelnen Individuen sehr erheblich. Sie betrugen bei Helianthus zwischen 18 und $29 \mathrm{mg} / \mathrm{g}$ Frischgewicht für die oberen, zwischen 12,5 und $22 \mathrm{mg}$ für die mittleren und $8 \mathrm{bis} 14 \mathrm{mg}$ für die unteren Sproßteile, bei Bryophyllum 5,6-9 mg für die oberen, 6-11 für die mittleren und 8-14 für die unteren Abschnitte. Aus diesen Zahlen ist ersichtlich, $\mathrm{da}$ die Sprosse von Helianthus absolut zuckerreicher sind als die von Bryophyllum, und vor allem auch, daß das Zuckergefälle bei beiden untersuchten Pflanzen entgegengesetzt ausgebildet ist.

Die großen Schwankungen der Absolutwerte machen es unmöglich, festzustellen, ob die Gesamtzuckermenge der gereizten Sprosse gesetzmäßige Unter- 
schiede gegenüber derjenigen aufrechter Organe aufweist. Auch muß vorläufig offen bleiben, auf welche Weise die Zuckerdifferenzen zustande kommen.

3. Der Sauerstoffuerbrauch der Oberund Unterseite geotropisch gereizter Sprosse

Wie oben erwähnt, wurde schon von Schley ${ }^{9}$ versucht, Atmungsdifferenzen zwischen den verschiedenen Flanken geotropisch gereizter Organe festzustellen; sie spaltete zu diesem Zwecke Vicia fabaSprosse nach der Reizung und hängte die Hälften in ein feuchte Kammer, die etwas $\mathrm{Ba}(\mathrm{OH})_{2}$ enthielt. Aus der Trübung der Lauge stellte sie dann durch bloßen Augenschein die Menge des ausgeschiedenen Kohlendioxyds fest und erhielt höhere Werte für die Unterbzw. Konvexseite. Da keinerlei Gewichtsvergleich angegeben wurde, konnten diese Experimente nicht als beweisend angesehen werden ${ }^{19}$.

Einen Hinweis auf einen engeren Zusammenhang zwischen geotropischer Reaktion und Atmungsstoffwechsel gaben auch die Untersuchungen von $\mathrm{Nav} \mathrm{e} \mathrm{z}^{20}$, der bei der Prüfung der Temperaturabhängigkeit der $\mathrm{CO}_{2}$-Abgabe und der geotropischen Reaktionszeit von Vicia faba-Keimlingen fand, daß der Temperaturkoeffizient für beide Größen denselben Wert hatte.

Die manometrischen Messungen des Sauerstoffverbrauches, die zum Zwecke einer eindeutigen Klärung der Frage unternommen wurden, zeigten nun, daß durchweg gesetzmäßige Differenzen auftreten, wobei die Unter- bzw. Konvexflanken der oberen Abschnitte des geotropisch gereizten Sprosses während der Krümmung stets einen höheren Sauerstoffverbrauch aufweisen als die Ober- bzw. Konkavseiten. Die Unterschiede liegen dabei z. Tl. beträchtlich außerhalb der Fehlergrenze von $12 \%$, wie durch Vergleich des Sauerstoffverbrauches der Hälften von 12 ungereizten Sprossen festgestellt wurde.

Abb. 5 gibt die Differenzen im Sauerstoffverbrauch zwischen Ober- und Unterseite in Prozenten des jeweiligen Mittelwertes der beiden Flanken und im Durchschnitt der ersten 6 Stdn. nach der Halbierung für verschiedene Zonen eines gereizten HelianthusSprosses wieder. Die Kardinalpunkte der Kurve für die Krümmungszone stimmen ausgezeichnet mit denen der entsprechenden Zuckerdifferenzkurve überein. Auch hier tritt der erste faßbare Unterschied nach $3 / 4$ Stdn. auf; das Maximum liegt bei etwa $12 \mathrm{Stdn}$.

19 H. F it ting, Z. Bot. 13, 404 [1921].

20 E. A. N a vez, J. gen. Physiol. 12, 641 [1929]. und die Differenzen verschwinden nach etwa $27 \mathrm{Stdn}$. In den dem Krümmungsabschnitt benachbarten, horizontal bleibenden Teilen des Sprosses (jeweils etwa $6 \mathrm{~cm}$ ) ist die Verschiebung im Sauerstoffverbrauch ebenfalls noch feststellbar. Allerdings liegen hier Beginn, Maximum und Ende der Unterschiede später und ihre Werte sind niedriger. Die untere Horizontal-

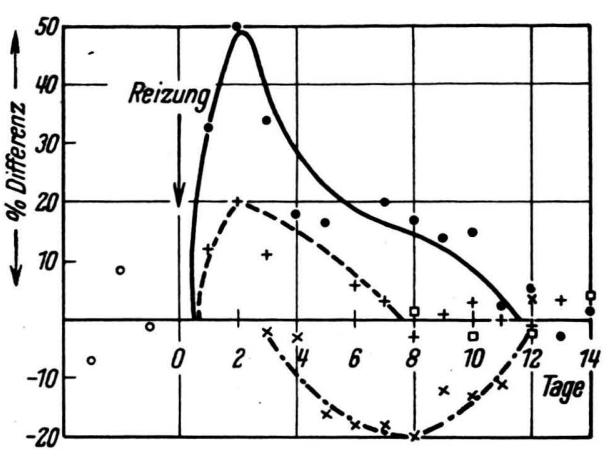

Abb. 6. Die Differenz im Sauerstoffverbrauch zwischen der Unter- bzw. Konvex- und der Ober- bzw. Konkavseite bei verschiedenen Abschnitten geotropisch gereizter Bryophyllum-Sprosse im Durchschnitt der ersten 6 Stdn. nach der Halbierung und in Prozenten des jeweiligen Mittelwertes

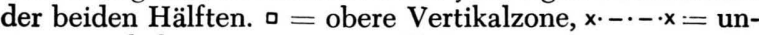
tere Vertikalzone, $\bullet-\bullet=$ Krümmungszone, +- - - + $=$ obere Horizontalzone, $0=$ Differenz zwischen den Hälften ungereizter Sprosse.

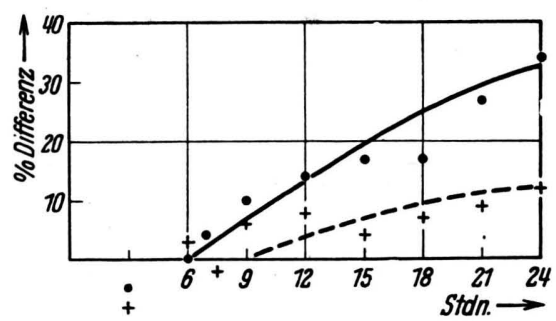

Abb. 7. Der Beginn der Ausbildung der Sauerstoffverbrauchs-Differenz zwischen den Hälften verschiedener Abschnitte geotropisch gereizter Bryophyllum-Sprosse.

$\bullet-\bullet=$ Krümmungszone, $-\cdots-+=$ oberer Horizontalabschnitt.

zone weist zwar auch auf der Unterseite noch vermehrten Sauerstoffverbrauch auf, doch bewegen sich die Werte durchweg innerhalb der Fehlergrenze. In den vertikalen Teilen des gekrümmten Sprosses ist in dem der Krümmungszone benachbarten Abschnitt überraschenderweise der Sauerstoffverbrauch der „Konkav“-Flanke höher; hier führt offenbar der Ausgleich der Wachstumsintensität der beiden Seiten vorübergehend $\mathrm{zu}$ einem intensiveren Stoffwechsel der aufholenden Hälfte. In den obèren Vertikalteilen läßt sich kein gesetzmäßiger, außerhalb der 
Fehlergrenze liegender Unterschied mehr erkennen.

Bei der Krümmungszone von Bryophyllum fallen Beginn und Ende der Differenzen im Sauerstoffverbrauch ebenfalls mit den entsprechenden Zeiten für die Zuckerunterschiede zusammen, während das Maximum bereits nach 2 Tagen, also erheblich früher, erreicht wird (Abb. 6 und 7). Auch bei diesem Objekt werden die anschließenden Horizontalzonen noch von dieser „Polarisierung“ der Atmung erfaßt, allerdings in wesentlich geringerem Ausmaß und für weit geringere Zeit als der gekrümmte Abschnitt. Die Vertikalzone zeigt in ihrem unteren Teil wie bei Helianthus eine gesetzmäßig ausgebildete, inverse Differenz im Sauerstoffverbrauch, die etwa gleichzeitig mit den Unterschieden im Krümmungsabschnitt ausgeglichen wird. In der oberen Vertikalzone endlich ist auch bei Bryophyllum die „Symmetrie“ im Stoffwechsel wiederhergestellt.

Die Absolutwerte des Sauerstoffverbrauches (in $\mathrm{mm}^{3} /$ Stde. und $\mathrm{mg}$ Trockengewicht) betrugen bei Helianthus für die jeweils oberste Sproßzone zwischen 2,46 und 5,0, für die anschließende zwischen 1,27 und 2,72 und für die nächstuntere zwischen 1,09 und 2,48; bei Bryophyllum zwischen 0,848 und 1,78 für die Spitzenteile, zwischen 0,42 und 1,427 für die mittleren und zwischen 0,38 und 1,17 für die unteren Abschnitte. Die starken Schwankungen erklären sich vor allem durch die verschiedene Länge der Dunkelreizung; bei längerem Assimilationsausfall nimmt naturgemäß die Atmung erheblich ab. Immerhin waren die Werte aber auch bei gleich behandelten Pflanzen so verschieden, daß die Frage, ob die gereizten Sprosse insgesamt einen gegenüber ungereizten veränderten Sauerstoffverbrauch aufweisen, nicht entschieden werden konnte.

Deutlich wird aus den Absolutwerten des Sauerstoffverbrauches aber, daß die „Verwässerung“ des Systems bei dem sukkulenten Bryophyllum neben einer verlängerten Reaktionszeit und einer Verminderung des absoluten Zuckergehaltes auch eine Herabsetzung der oxydativen Prozesse zur Folge hat.

Die wichtige Frage, ob die verstärkte Sauerstoffaufnahme als eine Intensivierung der Atmung betrachtet werden kann, war am einfachsten durch die Ermittelung des respiratorischen Quotienten $R Q$, also des Verhältnisses $\mathrm{CO}_{2} / \mathrm{O}_{2}$, zu prüfen. Die Verwendung des vermehrt aufgenommenen Sauerstoffes zu oxydativen Prozessen, die nicht bis zur Bildung gasförmiger Endprodukte führten, mußte sich hier durch eine Verschiebung des Quotienten zu erkennen geben.
Die Bestimmung ergab für Helianthus im ungereizten Zustand 1,015, nach 12-stdg. Reizung 1,021, für vertikales Bryophyllum 0,988 und für 2 Tage horizontal gelegte Pflanzen 0,992 (jeweils Mittel von 3 Bestimmungen). Die $R Q$-Werte der Ober- und Unterseiten der gereizten Sprosse unterschieden sich kaum und nicht gesetzmäßig. Es kann demnach gesagt werden, daß die verstärkte $\mathrm{CO}_{2}$-Aufnahme geotropisch gereizter Organe einer Steigerung der Atmung dient.

\section{Der Zusammenhang zwischen den}

A t mungs - und Zuckerdifferenzen

Die Tatsache, daß die Verschiebungen im Zuckergehalt und in der Atmungsintensität etwa gleichzeitig auftreten und ungefähr gleichlange wahrnehmbar sind, legte zunächst die Vermutung nahe, daß beide Prozesse eng miteinander zusammenhängen und die Steigerung der Sauerstoffaufnahme durch den höheren Zuckergehalt bedingt sei. Dieser Annahme widersprach jedoch die Beobachtung, daß sich die Unterschiede in der Atmungsintensität der getrennten Sproßhälften auch nach $20 \mathrm{Stdn}$. noch kaum verringert hatten, nach einer Zeit also, in der sich die ursprüngliche Zuckerdifferenz längst hätte ausgleichen müssen.

Zur weiteren Klärung dieser Frage wurden die Ober- und Unterhälften gereizter Helianthus- und Bryophyllum-Sprosse mit einer Rasierklinge in feinste Scheiben zerschnitten und so in dem üblichen $1 / 45^{-}-\mathrm{mol}$. Phosphatpuffer suspendiert, dem aber nach einer 1-stdg. Leeratmung eine Glucoselösung der Stärke zugesetzt wurde, daß die Endkonzentration der Versuchslösung 2\% betrug. Damit wird das Maximum der durch Zuckerzusatz erreichbaren Atmungssteigerung, das bei ungereizten Sprossen der untersuchten Pflanzen bei einer Konzentration von $1-1,5 \%$ erreicht wird, sicher gewährleistet. Hing nun die Differenz der Sauerstoffaufnahme zwischen der Unterund Oberseite allein von der unterschiedlichen Menge des verfügbaren Zuckers ab, so mußte sie unter diesen Bedingungen verschwinden. Der Versuch ergab, daß bei einen Tag gereizten Bryophyllum-Pflanzen nach 4 Stdn. die Differenz im Sauerstoffverbrauch der Hälften der Krümmungszone von ursprünglich $31,6 \%$ auf $26,4 \%$ und bei dem anschließenden Horizontalabschnitt von 10,0 auf $8,3 \%$ gefallen war, dieser Unterschied dann aber während der gesamten Versuchszeit von $12 \mathrm{Stdn}$. konstant erhalten blieb. Die Atmungssteigerung nach dem Glucosezusatz betrug für die Unterseite etwa $20 \%$, für diè Ober- 
seite gegen $30 \%$ bei Absolutwerten von 0,983 bis $1,66 \mathrm{~mm}^{3} /$ Stde. und $\mathrm{mg}$ Trockengewicht für die Krümmungszone und von 0,87 bis $1,13 \mathrm{~mm}^{3}$ für den Horizontalabschnitt.

Auch bei Helianthus zeigt sich bei Glucosezusatz ein leichter Rückgang der durch geotropische Reizung induzierten Atmungsdifferenz, jedoch kommt es auch hier nicht zu einem Ausgleich der Unterschiede.

Es kann damit als erwiesen gelten, daß die Differenzen im Sauerstoffverbrauch der Ober- und Unter- bzw. Konkav- und Konvexseite geotropisch gereizter Sprosse nur zu einem geringen Teil auf die verschiedene Menge des verfügbaren Zuckers zurückzuführen ist, in der Hauptsache aber auf Ursachen zurückgeht, die noch der Klärung bedürfen.

Ein Wort noch zu der Wirkung der verschiedenen Zerteilung auf die Sauerstoffaufnahme der verwendeten Sprosse. Zunehmend starke Zerkleinerung hat bis zu der sog. Grenzschnittdicke infolge der erleichterten Sauerstoffversorgung der inneren Gewebeteile eine Steigerung des Sauerstoffverbrauches zur Folge; diese betrug z. B. bei einer mit einer Rasierklinge feinst zerteilten Bryophyllum-Sproßhälfte $11 \%$ gegenüber derselben Trockengewichtsmenge der anderen Hälfte, die in $3 \mathrm{~cm}$ langen Stücken für den Versuch verwendet worden war. Von Wichtigkeit für unsere Betrachtung ist nun der Einfluß der durch die Zerteilung bewirkten Verstärkung der Sauerstoffaufnahme auf die durch die Reizung induzierte Differenz der Atmungsintensität. Entsprechende Beobachtungen zeigten, daß die Unterschiede im Sauerstoffverbrauch der Unter- und Oberseite geotropisch gereizter Sprosse von Bryophyllum und Helianthus bei den verschiedensten Zerteilungsgraden stets in derselben Richtung und in konstanter Stärke ausgebildet waren.

\section{Besprechung der Ergebnisse}

Es dürfte außer Zweifel stehen, daß die infolge der geischen Reizung auftretenden Unterschiede in der Atmungsintensität der Ober- und Unterseiten von Sprossen aufs engste mit der tropistischen Reaktion

21 B. Commoner u. K. V. Thimann, J. gen. Physiol. 24, 279 [1941].

$22 \mathrm{~K}$. V. Thimann u. W. D. B o n n e r jr., Biologic. Bull. 96, 296 [1949]. verknüpft sind. Das Problem ist nun das Zustandekommen dieser Differenz, vor allem der mögliche Zusammenhang mit der Wuchsstoffverschiebung. Es wäre durchaus denkbar, daß der vermehrte Gehalt an Indolylessigsäure der Unter- bzw. Konvexseite verantwortlich zu machen ist für die Intensivierung der oxydativen Prozesse, da nach zahlreichen Untersuchungen (u. a. Commoner und Thimann ${ }^{21}$, $\mathrm{Th}$ imann und $\mathrm{B}$ on $\mathrm{ner}{ }^{22}, \mathrm{~K}$ andler und Vieregg ${ }^{23}$ ) der Wuchsstoff auch bei der Zugabe von außen eine derartige Wirkung entfaltet.

In dieses Bild würde auch die von $\mathrm{G}$ e $\beta \mathrm{n}$ e r ${ }^{24}$ bei Myriophyllum-Sprossen festgestellte Tatsache passen, daß die Gesamtatmung der Organe bei geotropischer Reizung nicht verändert wird. Es käme die Differenz dann so zustande, daß der Sauerstoffverbrauch der stärker wachsenden Seite um ebensoviel über dem Normalwert liegt wie derjenige der schwächer wachsenden Flanke darunter. Die Analogie zu dem Wuchsstoffgehalt liegt auf der Hand. Allerdings bedarf die Vermutung, daß bei Helianthus und Bryophyllum die Gesamtatmung durch die Reizung ebenfalls nicht beeinflußt wird, noch der experimentellen Bestätigung; entsprechende Versuche sind im Gange.

Die gleichzeitig mit den Atmungsunterschieden auftretenden Zuckerdifferenzen, die weder die entscheidende Ursache noch die Folge des verschiedenen Sauerstoffverbrauches sind, weisen darauf hin, daß die durch die geische Reizung induzierte physiologische Dorsiventralität mit der „Polarisierung“ der Atmung nicht vollständig erfaßt ist. Es liegt nahe anzunehmen, daß nicht nur das Atmungsferment, dem die Indolylessigsäure angehört, in seiner Menge und Aktivität beeinflußt wird, sondern ebenso andere Enzyme, vor allem die Amylase. Entsprechende Untersuchungen sind auch hier eingeleitet.

Einen unmittelbaren Ansatzpunkt zur Klärung des reizphysiologischen Zentralproblems, nämlich der Frage nach den Primärwirkungen des Reizes, geben die geschilderten Ergebnisse noch nicht; immerhin dürfte es möglich sein, auf dem eingeschlagenen Wege auch hier zu einer präziseren Fragestellung zu kommen.

23 Noch unveröffentlicht; nach frdl. Mitteilung.

24 Unveröffentlicht; nach frdl. Mitteilung. 